\title{
Comprehensive analysis of genomic alterations detected by next-generation sequencing-based tissue and circulating tumor DNA assays in Chinese patients with non-small cell lung cancer
}

\author{
HUA YANG $^{1}$, JUNJIE ZHANG ${ }^{2}$, LEMENG ZHANG ${ }^{1}$, XIAOPING WEN $^{1}$, YONGZHONG LUO $^{1}$, \\ DINGQUAN YAO ${ }^{1}$, TIANLI CHENG $^{1}$, HUANQING CHENG $^{3}$, HUINA WANG $^{3}$, FENG LOU $^{3}$, \\ JING GUO $^{3}$, XIAYUAN LIANG ${ }^{3}$, SHANBO CAO $^{3}$ and JIANHUA CHEN ${ }^{1}$
}

\begin{abstract}
${ }^{1}$ Department of Thoracic Internal Medicine, Hunan Cancer Hospital, Affiliated Cancer Hospital of Xiangya Medical School of Central South University, Changsha, Hunan 410013; ${ }^{2}$ Department of Anesthesiology, Xiangya Hospital, Central South University, Changsha, Hunan 410008; ${ }^{3}$ Acornmed Biotechnology Co., Ltd., Beijing 100176, P.R. China
\end{abstract}

Received August 15, 2018; Accepted July 3, 2019

DOI: $10.3892 / \mathrm{ol} .2019 .10791$

\begin{abstract}
While tumor genotyping is the standard treatment for patients with non-small cell lung cancer (NSCLC), spatial and temporal tumor heterogeneity and insufficient specimens can lead to limitations in the use of tissue-based sequencing. Circulating tumor DNA (ctDNA) fully encompasses tumor-specific sequence alterations and offers an alternative to tissue sample biopsies. However, few studies have evaluated whether the frequency of multiple genomic alterations observed following ctDNA sequencing is similar to that observed following tissue sequencing in NSCLC. Therefore, in the present study, targeted next-generation sequencing (NGS) was performed on tissue and plasma ctDNA samples in 99 patients with NSCLC. Overall, the frequencies of genetic alterations detected in ctDNA were positively correlated with those detected via tissue profiling $(\mathrm{r}=0.812 ; \mathrm{P}=0.022)$. Genomic data revealed significant mutual exclusivity between alterations in epidermal growth factor receptor (EGFR) and tumor protein 53 (TP53; $\mathrm{P}=0.020$ ), and between alterations in EGFR and KRAS ( $\mathrm{P}=0.008)$, as well as potential mutual exclusivity between alterations in EGFR and Erb-B2 receptor tyrosine kinase $2(\mathrm{P}=0.059)$. Furthermore, the EGFR mutant allele frequency (MAF) was positively correlated with the TP53 MAF in individual tumors $(\mathrm{r}=0.773 ; \mathrm{P}=0.005)$, and there
\end{abstract}

Correspondence to: Dr Jianhua Chen, Department of Thoracic Internal Medicine, Hunan Cancer Hospital, Affiliated Cancer Hospital of Xiangya Medical School of Central South University, 283 Tongzipo Road, Changsha, Hunan 410013, P.R. China

E-mail: chenjianhua@hnszlyy.com

Dr Shanbo Cao, Acornmed Biotechnology Co., Ltd., 13 Kechuang Road, Beijing 100176, P.R. China

E-mail: shanbocao@acornmed.com

Key words: circulating tumor DNA, tissue, non-small cell lung cancer, next-generation sequencing, targeted therapy was a marked difference in the EGFR MAF between patients with and without the TP53 mutation $(\mathrm{P}=0.001)$. Levels of the tumor serum marker CA242 in patients with ctDNA-detectable mutations were higher compared with those in patients without ctDNA-detectable mutations. The data from the present study highlight the importance of tissue and plasma ctDNA screening by NGS to guide personalized therapy and promote the clinical management of patients with NSCLC.

\section{Introduction}

Non-small cell lung cancer (NSCLC) is a leading cause of cancer-associated mortality worldwide, accounting for $\sim 85 \%$ of lung cancer cases $(1,2)$. An estimated 1.8 million new lung cancer cases and 1.6 million lung cancer-associated mortalities were reported worldwide in 2012 (3). Advances in genomic research and targeted therapy have greatly improved the development of therapeutic strategies and the clinical outcomes of patients with NSCLC $(4,5)$. Activation of specific tyrosine kinases, including those derived from epidermal growth factor receptor (EGFR) mutations or the rearrangement of the anaplastic lymphoma kinase (ALK) gene, promotes development in the corresponding molecular inhibitors in EGFR mutation and ALK translocation positive subgroups (6-8). In addition to EGFR and ALK, a number of other oncogenic driver genes, such as ROS1, BRAF, MET, RET and Erb-B2 receptor tyrosine kinase 2 (ERBB2) have been identified as relevant targets in NSCLC (9).

Next-generation sequencing (NGS) has led to considerable advances in the comprehensive analysis of genomic alterations in cancer research and clinical application $(10,11)$. At present, the detection of genomic alterations in tumors is primarily reliant on cancer tissue analysis. Despite the benefits and therapeutic insights offered by tissue sample testing, spatial and temporal tumor heterogeneity, and insufficient and unacquirable specimens can lead to limitations in the use of tissue-based sequencing $(12,13)$. Circulating tumor DNA (ctDNA), released into the plasma from apoptotic and necrotic tumor cells derived from primary tumors and metastatic lesions, are comprised of 
tumor-specific sequence alterations and may be an alternative target to tissue sample biopsies (14-16). An increasing body of evidence has indicated that ctDNA testing could be utilized for patients without acquirable tissue samples to screen for genetic variations and thus guide treatment decisions in patients with NSCLC (17-19).

Despite the increasing utilization of plasma ctDNA mutations in guiding clinical decisions, few studies have evaluated whether the frequency of multiple genomic alterations observed following ctDNA sequencing is similar to that observed following tissue sequencing in NSCLC $(17,20)$. Therefore, in order to investigate the associations in the frequency of comprehensive genomic alterations between ctDNA and tissue profiling in Chinese patients with NSCLC, the present study analyzed 59 plasma ctDNA samples and 40 tissue samples via targeted NGS. The results provide important insights into the potential benefits of ctDNA profiling in patients with NSCLC in clinical practice.

\section{Materials and methods}

Patients and sample collection. The present study enrolled 99 patients with NSCLC who were baseline or previously treated at the Hunan Cancer Hospital (Changsha, China) between September 2017 and April 2018. The specimens included 59 plasma and 40 tissue samples. The inclusion criteria for the patients were as follows: i) Patients were diagnosed with NSCLC by histopathology or cytology; ii) tissue specimens were confirmed by qualified pathologists, from Hunan Cancer Hospital who were blinded to the study and ensured $>30 \%$ tumor content; and iii) at least $150 \mathrm{ng}$ and 15 ng DNA from each tissue and ctDNA sample, respectively, were successfully extracted. Patients who had other malignant tumors and serious mental illness were excluded. Written informed consent was provided by all participants. All experiments were approved by and performed in accordance with the relevant guidelines and regulations of the Committee of Medical Ethics of Hunan Cancer Hospital.

Targeted deep sequencing. ctDNA was isolated from $\geq 2 \mathrm{ml}$ plasma with a QIAamp Circulating Nucleic Acid kit (Qiagen $\mathrm{GmbH}$ ) according to the manufacturer's protocol. Tissue DNA was extracted using the QIAamp Genomic DNA kit (Qiagen $\mathrm{GmbH}$ ). The quality of the DNA and DNA quantification were assessed using the Agilent 2100 BioAnalyzer (Agilent Technologies, Inc.) and Qubit ds DNA HS assay kit (Thermo Fisher Scientific, Inc.).

Sequencing libraries were constructed according to the Illumina standard library construction instructions (Illumina, Inc.). Genomic DNA extracted from tissue samples was first sheared to $250 \mathrm{bp}$ with an ultrasonoscope. The fragmented DNA was subjected to end-repairing, A-tailing and ligation to the adapters with barcode sequences. Subsequently, PCR was performed and the resulting products were purified with AMPure XP magnetic beads (Agencourt AMPure XP kit; Beckman Coulter, Inc.) according to the manufacturer's protocol. The various libraries were then hybridized with a nine-gene panel, which was enriched for the coding regions and selected introns of genes with known relevance to NSCLC. The target-enriched libraries were then pooled and sequenced on an Illumina HiSeq2500 NGS platform (Illumina, Inc.). The sequencing depth was $>10,000 x$. Reads were aligned to the human genomic reference sequences (hg19) using the Burrows-Wheeler alignment (BWA) tool (21). Local realignment and base quality score recalibration were conducted using GATK software (version 2.3; software.broadinstitute. org/gatk) (22). MuTect2 (version 1.1.1; software.broadinstitute. org/cancer/cga/mutect) with the recommended parameters was used to identify single-nucleotide variants (SNVs) and small insertions or deletions (INDELs) (23). Copy number variants (CNV) calling was performed with CONTRA software (version 2.0.4; contra-cnv.sourceforge.net) (24).

The cancer genome Atlas (TCGA) datasets. Multiplatform genomics data was previously published in The Cancer Genome Atlas (TCGA; cancergenome.nih.gov). In total, 1,144 NSCLC cases comprising 660 patients with lung adenocarcinoma (AD) and 484 patients with lung squamous cell carcinoma (SC) were included in TCGA cohort with gene mutation data (25).

Detection of tumor serum markers. Tumor serum markers were detected by a C12 multi-tumor marker protein chip system (Shanghai HealthDigit Co., Ltd.). Based on the manufacturer's instruction, $100 \mu \mathrm{l}$ of the different calibrators, quantitative quality control products and serum specimen were transferred to the reaction well of the chip, which was subsequently incubated at $37^{\circ} \mathrm{C}$ for $30 \mathrm{~min}$ with agitation. The mixture was discarded and the well was washed four times with the wash solution. Next, $100 \mu$ l reaction solution was added to the well and incubated at $37^{\circ} \mathrm{C}$ for $30 \mathrm{~min}$ with agitation. The mixture was discarded and the well was washed four times with the wash solution. Subsequently, detection solution $\mathrm{A}$ and $\mathrm{B}$ were added to the surface of the chip and incubated at room temperature for $1.5 \mathrm{~min}$. The chip was placed into the biochip scanner (HD-2001A; Shanghai HealthDigit Co., Ltd.) to analyze each tumor serum marker.

Statistical analysis. SPSS statistical software (version 21.0; IBM Corp.) was used to analyze the data. Differences in continuous variables were assessed using unpaired Student's t-test. Associations between different genotypes and clinical characteristics were analyzed using Fisher's exact test or $\chi^{2}$ test. Correlations between variables were assessed using Spearman's rank correlation coefficient. Patterns of co-alteration or mutual exclusivity between EGFR and other driver genes were analyzed by Fisher's exact test. The odds ratio (OR) is a statistic that quantifies the strength of the association between two events. In the current study, EGFR mutations and other driver gene mutations were classified as events $\mathrm{A}$ and $\mathrm{B}$, respectively. The odds ratio was calculated as the ratio of the odds of $\mathrm{A}$ in the presence of $\mathrm{B}$ and the odds of $\mathrm{A}$ in the absence of $\mathrm{B} . \mathrm{P}<0.05$ was considered to indicate a statistically significant result.

\section{Results}

NGS-based tissue and ctDNA assays to identify genomic alterations among 99 patients with NSCLC. In the present study, a total of 99 patients diagnosed with NSCLC were 
Table I. Clinicopathological characteristics among 99 patients with non-small cell lung cancer.

\begin{tabular}{|c|c|c|c|c|}
\hline Characteristic & Total & Tissue, $\mathrm{n}=40$ & ctDNA, $n=59$ & P-value \\
\hline Age, years, median (range) & $56(39-83)$ & $56(46-77)$ & $58(39-83)$ & 0.534 \\
\hline $\operatorname{Sex}, \mathrm{n}(\%)$ & & & & 0.112 \\
\hline Male & 73 (73.7) & $26(65.0)$ & $47(79.7)$ & \\
\hline Female & $26(26.3)$ & $14(35.0)$ & $12(20.3)$ & \\
\hline Smoking status, $\mathrm{n}(\%)$ & & & & 0.068 \\
\hline Yes & 64 (64.6) & $24(60.0)$ & $40(67.8)$ & \\
\hline No & $27(27.3)$ & $15(37.5)$ & $12(20.3)$ & \\
\hline Unknown & $8(8.1)$ & $1(2.5)$ & $7(11.9)$ & \\
\hline Histological type, n (\%) & & & & 0.195 \\
\hline $\mathrm{AD}$ & $58(58.6)$ & $28(70.0)$ & $30(50.8)$ & \\
\hline $\mathrm{SC}$ & $36(36.4)$ & $10(25.0)$ & $26(44.1)$ & \\
\hline LCC & $1(1.0)$ & $0(0.0)$ & $1(1.7)$ & \\
\hline ASC & $4(4.0)$ & $2(5.0)$ & $2(3.4)$ & \\
\hline Clinical stage, n (\%) & & & & 0.417 \\
\hline I & $1(1.0)$ & $0(0.0)$ & $1(1.7)$ & \\
\hline II & $3(3.0)$ & $2(5.0)$ & $1(1.7)$ & \\
\hline III & $19(19.2)$ & $6(15.0)$ & $13(22.0)$ & \\
\hline IV & $67(67.7)$ & $30(75.0)$ & $37(62.7)$ & \\
\hline Unknown & $9(9.1)$ & $2(5.0)$ & 7 (11.9) & \\
\hline
\end{tabular}

AD, lung adenocarcinoma; SC, lung squamous cell carcinoma; LCC, large cell carcinoma; ASC, adenosquamous carcinoma; ctDNA, circulating tumor DNA.

enrolled. NGS was performed for specimens, including 59 plasma ctDNA and 40 tissue samples. Among the patients, the median age at diagnosis was 56 years (range, 39-83 years), $73.7 \%$ of the patients were male, $64.6 \%$ of the patients were smokers, $58.6 \%$ of the patients were diagnosed with AD, and $67.7 \%$ of the patients had stage IV cancer. The clinical and pathological characteristics of the patients are listed in Table I.

All 99 samples were profiled by targeted sequencing with a panel of nine of the most common driver genes [EGFR, ALK, ROS1, BRAF, MET, RET, ERBB2, KRAS and tumor protein 53 (TP53)] in NSCLC. A total of 38 out of 40 (95.0\%) tissue samples and 38 out of $59(64.4 \%)$ ctDNA samples exhibited at least one genetic alteration. TP53 was the most commonly mutated gene (56.6\%), followed by EGFR (36.8\%). Compared with TCGA data, significantly more somatic mutations were observed in EGFR and ERBB2 $(\mathrm{P}<0.001)$, and significantly fewer mutations in TP53 ( $\mathrm{P}=0.045)$ were identified among the cohort of Chinese patients with NSCLC (Table II). Although the KRAS mutation rate according to TCGA was higher than that of the cohort in the present study, no significant difference was observed $(\mathrm{P}=0.179$; Table II). Further analysis demonstrated that the frequency of TP53 alterations was markedly higher in $\mathrm{SC}$ than in $\mathrm{AD}(\mathrm{P}=0.009$; Table III), and the frequency of EGFR alterations was associated with being female, $\mathrm{AD}$ and non-smokers $(\mathrm{P}<0.001, \mathrm{P}=0.001$ and $\mathrm{P}<0.001$, respectively; Table III). KRAS alterations were more likely to occur in male patients and smokers, although no statistically significant differences were observed $(\mathrm{P}=0.086$ and $\mathrm{P}=0.105$, respectively). ERBB2 alterations were more frequently
Table II. Frequency of somatic mutations among patients with non-small cell lung carcinoma in the present cohort and TCGA cohort.

\begin{tabular}{lccr}
\hline & \multicolumn{3}{c}{ Frequency, } \\
\cline { 2 - 3 } Somatic \\
\cline { 2 - 3 } mutations & $\begin{array}{c}\text { TCGA } \\
(\mathrm{n}=1,144)\end{array}$ & $\begin{array}{c}\text { The present } \\
\text { cohort }(\mathrm{n}=76)\end{array}$ & P-value \\
\hline TP53 & 67.7 & 56.6 & 0.045 \\
EGFR & 10.2 & 36.8 & $<0.001$ \\
KRAS & 19.4 & 13.2 & 0.179 \\
ERBB2 & 2.3 & 9.2 & $<0.001$ \\
BRAF & 6.1 & 5.3 & 0.762 \\
MET & 3.0 & 5.3 & 0.267 \\
\hline
\end{tabular}

TCGA, The Cancer Genome Atlas; TP53, tumor protein 53; EGFR, epidermal growth factor receptor; KRAS, Kirsten rat sacoma viral oncogene homolog; ERBB2, Erb-B2 receptr tyrosine kinase; BRAF, v-raf murine sarcoma viral oncogene homolog B1; MET, mesenchymal-epithelial transition factor.

observed in non-smokers $(\mathrm{P}<0.001)$. Furthermore, in the present study, no ERBB2 alterations were observed in patients with localized NSCLC, and statistical analysis revealed that ERBB2 alterations were potentially associated with metastatic stage in NSCLC ( $\mathrm{P}=0.108$; Table III). 
Table III. Analysis between genetic alterations and clinicopathological characteristics.

\begin{tabular}{|c|c|c|c|c|c|c|c|c|}
\hline \multirow[b]{2}{*}{ Characteristic } & \multicolumn{2}{|c|}{ TP53 } & \multicolumn{2}{|c|}{ EGFR } & \multicolumn{2}{|c|}{ KRAS } & \multicolumn{2}{|c|}{ ERBB2 } \\
\hline & Freq, $\%$ & P-value & Freq, \% & P-value & Freq, $\%$ & P-value & Freq, $\%$ & P-value \\
\hline \multicolumn{9}{|l|}{ Sex } \\
\hline Male & 57.4 & 0.819 & 20.4 & $<0.001$ & 20.4 & 0.086 & 14.8 & 0.503 \\
\hline Female & 54.5 & & 77.3 & & 4.5 & & 9.1 & \\
\hline \multicolumn{9}{|l|}{ Age, years } \\
\hline$\leq 60$ & 52.5 & 0.532 & 45.0 & 0.061 & 10.0 & 0.129 & 10.0 & 0.410 \\
\hline$>60$ & 60.0 & & 23.3 & & 23.3 & & 16.7 & \\
\hline \multicolumn{9}{|c|}{ Histological type } \\
\hline $\mathrm{AD}$ & 46.8 & 0.009 & 48.9 & 0.001 & 14.9 & 0.684 & 12.8 & 0.804 \\
\hline $\mathrm{SC}$ & 77.8 & & 11.1 & & 18.5 & & 14.8 & \\
\hline \multicolumn{9}{|l|}{ Smoking } \\
\hline Yes & 58.0 & 0.663 & 20.0 & $<0.001$ & 20.0 & 0.105 & 2.0 & $<0.001$ \\
\hline No & 52.4 & & 71.4 & & 4.8 & & 38.1 & \\
\hline \multicolumn{9}{|l|}{ Clinical stage } \\
\hline $\mathrm{I}+\mathrm{II}+\mathrm{III}$ & 57.1 & 1.000 & 35.7 & 1.000 & 21.4 & 0.511 & 0.0 & 0.108 \\
\hline IV & 57.1 & & 35.7 & & 14.3 & & 16.1 & \\
\hline
\end{tabular}

TP53, tumor protein 53; EGFR, epidermal growth factor receptor; KRAS, Kirsten rat sarcoma viral oncogene homolog; ERBB2, Erb-B2 receptor tyrosine kinase; Freq, frequency of genomic alterations; AD, lung adenocarcinoma; SC, lung squamous cell carcinoma.

Overall, $50.5 \%(50 / 99)$ of the patients with NSCLC harbored at least one targetable alteration (data not shown). Regarding pathological type, the frequency of the targetable alterations between patients with AD (63.8\%) and SC (30.6\%) were significantly different $(\mathrm{P}=0.002$; Table IV). The landscape of genomic mutations demonstrated that an EGFR mutation was seen alongside each other gene (MET, ERBB2, KRAS and ALK) mutation in only one patient each (1\%). In addition, the present study demonstrated that EGFR amplification coexisted with EGFR mutations, including SNVs and INDELs, but was mutually exclusive with the EGFR T790M mutation. Furthermore, no patients with concurrent ERBB2 mutation and amplification were observed. All four patients that possessed the EGFR T790M mutation also harbored other EGFR mutations (Fig. 1). To investigate the patterns of concurrent alterations and mutual exclusivity, pairwise associations between somatic events were examined. Mutual exclusivity was observed between alterations in EGFR and TP53 [odds ratio $(\mathrm{OR})=0.324 ; \mathrm{P}=0.020$; Table V] and between those in EGFR and KRAS $(\mathrm{OR}=0.091 ; \mathrm{P}=0.008)$ in patients with NSCLC. Furthermore, potential mutual exclusivity between EGFR and ERBB2 alterations was also observed, but this was not statistically significant $(\mathrm{OR}=0.161$; $\mathrm{P}=0.059$; Table V).

Comparison of the frequency of genomic variants between tissue and ctDNA samples. In order to investigate the use of ctDNA sequencing, the present study compared the prevalence of genomic alterations, including non-synonymous SNVs, CNVs and rearrangements, between tissue and ctDNA samples. Overall, there were marked similarities in the frequency of variants when comparing the tissue and ctDNA
Table IV. Analysis of frequency of targetable alterations between $\mathrm{AD}$ and $\mathrm{SC}$.

\begin{tabular}{lcc}
\hline $\begin{array}{l}\text { Histological } \\
\text { type }\end{array}$ & $\begin{array}{c}\text { Frequency of targetable } \\
\text { alterations, \% }\end{array}$ & P-value \\
\hline AD & 63.8 & 0.002 \\
SC & 30.6 & \\
\hline
\end{tabular}

$\mathrm{AD}$, lung adenocarcinoma; SC, lung squamous cell carcinoma.

samples (Fig. 2A). Further analysis indicated that the prevalence of alterations among the most common NSCLC driver genes in ctDNA was positively correlated with that observed in tumor tissues ( $\mathrm{r}=0.812 ; \mathrm{P}=0.022$; Fig. $2 \mathrm{~B}$ ).

Association between EGFR and TP53 mutant allele frequencies (MAFs). Evolutionary studies of NSCLC have demonstrated that TP53 and EGFR mutations are the two most dominant clonal mutations (26); thus, the present study hypothesized that there may be a correlation between the MAFs of TP53 and EGFR in individual patients. As expected, a significantly linear relationship was observed $(r=0.773 ; P=0.005$; Fig. $3 \mathrm{~A})$. However, when the MAFs of TP53 and KRAS were compared, no linear correlation was identified ( $\mathrm{r}=0.700 ; \mathrm{P}=0.233$; Fig. $3 \mathrm{~B})$. In addition, the present study observed significant differences in the MAF of EGFR between patients with and without TP53 mutations $(\mathrm{P}=0.001$; Fig. $3 \mathrm{C})$. By contrast, no clear difference in the MAF of TP53 was observed between patients with and without EGFR mutations ( $\mathrm{P}=0.122$; Fig. 3D). 
Table V. Patterns of association between somatic events in non-small cell lung carcinoma.

\begin{tabular}{|c|c|c|c|c|c|c|}
\hline \multirow[b]{2}{*}{ EGFR } & \multicolumn{2}{|c|}{ TP53 } & \multicolumn{2}{|c|}{ KRAS } & \multicolumn{2}{|c|}{ ERBB2 } \\
\hline & OR & P-value & OR & $\mathrm{P}$-value & OR & P-value \\
\hline Statistical value & 0.324 & 0.020 & 0.091 & 0.008 & 0.161 & 0.059 \\
\hline
\end{tabular}

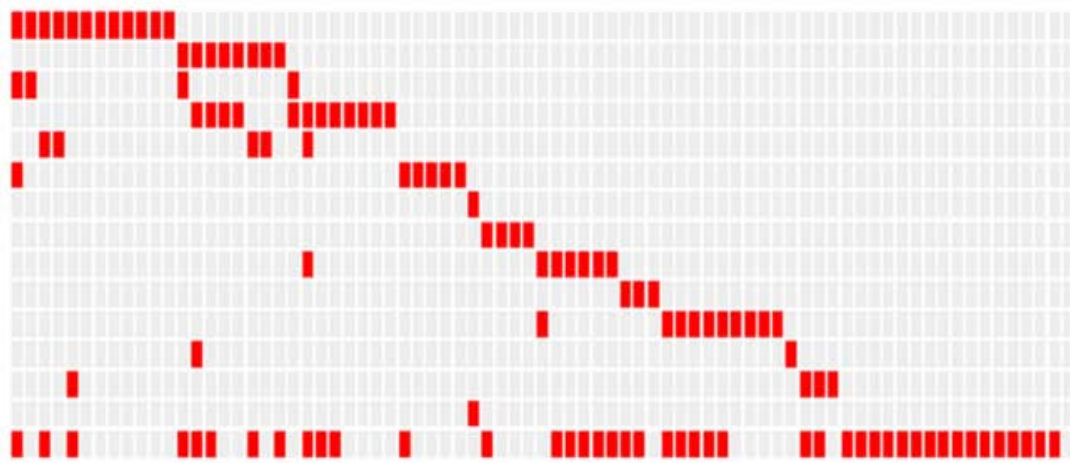

EGFR 19 del
EGFR $L 858 \mathrm{R}$
EGFR T790M
EGFR other snv/indel
EGFR amplification
ALK fusion
ALK amplification
BRAF snv/indel
ERBB2 snv/indel
ERBB2 amplification
KRAS snv/indel
KRAS amplification
MET snv/indel
$R E T$ fusion
TP53 snv/indel

Figure 1. Landscape of genomic alterations among 99 patients with non-small cell lung cancer. Red boxes indicate alterations. EGFR, epidermal growth factor receptor; del, small deletion; snv, single nucleotide variant; ALK, anaplastic lymphoma kinase; indel, small insertion or deletion; BRAF, v-raf murine sarcoma viral oncogene homolog B1; KRAS, Kirsten rat sarcoma viral oncogene homolog; MET, mesenchymal-epithelial transition factor; RET, rearranged during transfection; TP53, tumor protein 53.
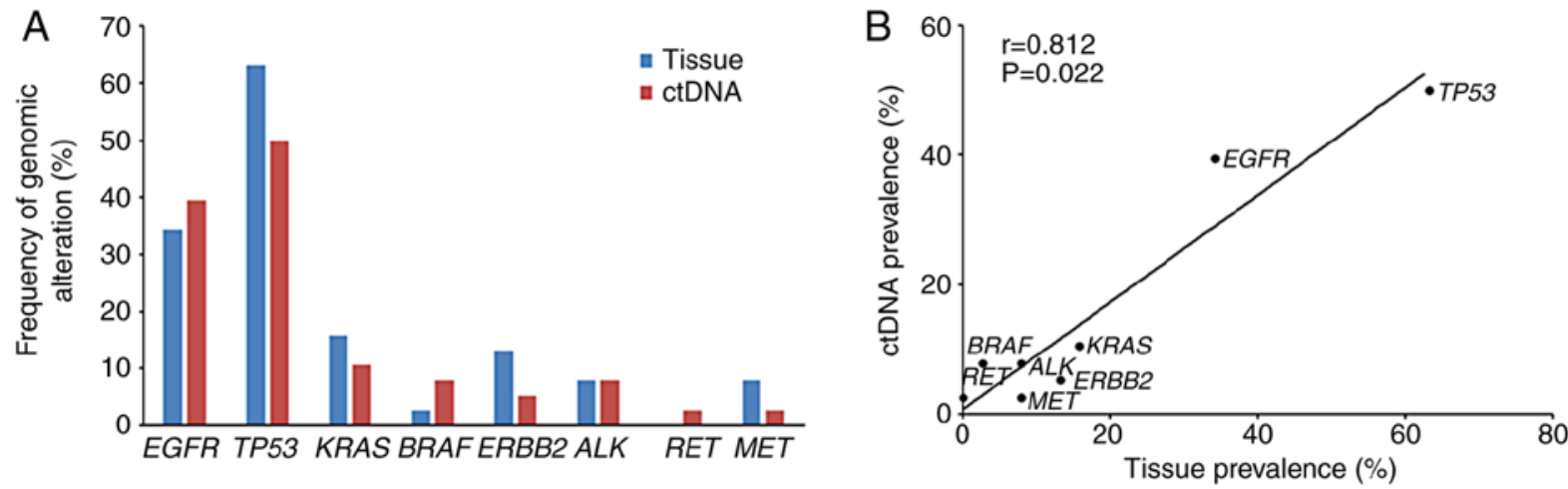

Figure 2. Genomic profiling of tissue and plasma ctDNA sequencing in patients with non-small cell lung cancer. (A) Comparison of gene alteration frequencies in tissue and plasma ctDNA samples. (B) Correlation analysis between gene alteration frequencies in ctDNA and tissue specimens. ctDNA, circulating tumor DNA; EGFR, epidermal growth factor receptor; TP53, tumor protein 53; KRAS, Kirsten rat sarcoma viral oncogene homolog; BRAF, v-raf murine sarcoma viral oncogene homolog B1; ERBB2, Erb-B2 receptor tyrosine kinase; ALK, anaplastic lymphoma kinase; RET, rearranged during transfection; MET, mesenchymal-epithelial transition factor.

Impact of the TP53 mutation on variant numbers in patients with NSCLC. An accumulating body of evidence has indicated that there may be an association between the number of variants (SNVs and INDELs) in baseline tumors and the clinical prognosis of lung cancer $(27,28)$. Furthermore, several studies have demonstrated that certain mutant genes, such as TP53 and KRAS, are associated with prognosis (29-31). Based on these discoveries, it was speculated that mutant genes may be associated with variant numbers in baseline tumors from patients with NSCLC. Therefore, the number of variants in each patient was analyzed in the context of various mutant genes. The results revealed that patients with TP53 mutations harbored more variant numbers when compared with patients without TP53 mutations in baseline tumors ( $\mathrm{P}=0.011$; Fig. 4). No other mutant genes were identified to be associated with variant numbers.

Association between ctDNA-detectable mutations and clinicopathological characteristics. Among the plasma samples, 21 out of 59 (35.6\%) samples exhibited no genomic alterations (data not shown). Based on this finding, the present study investigated the association between the frequency of ctDNA-detectable mutations and clinicopathological characteristics. The results revealed that the frequency of 

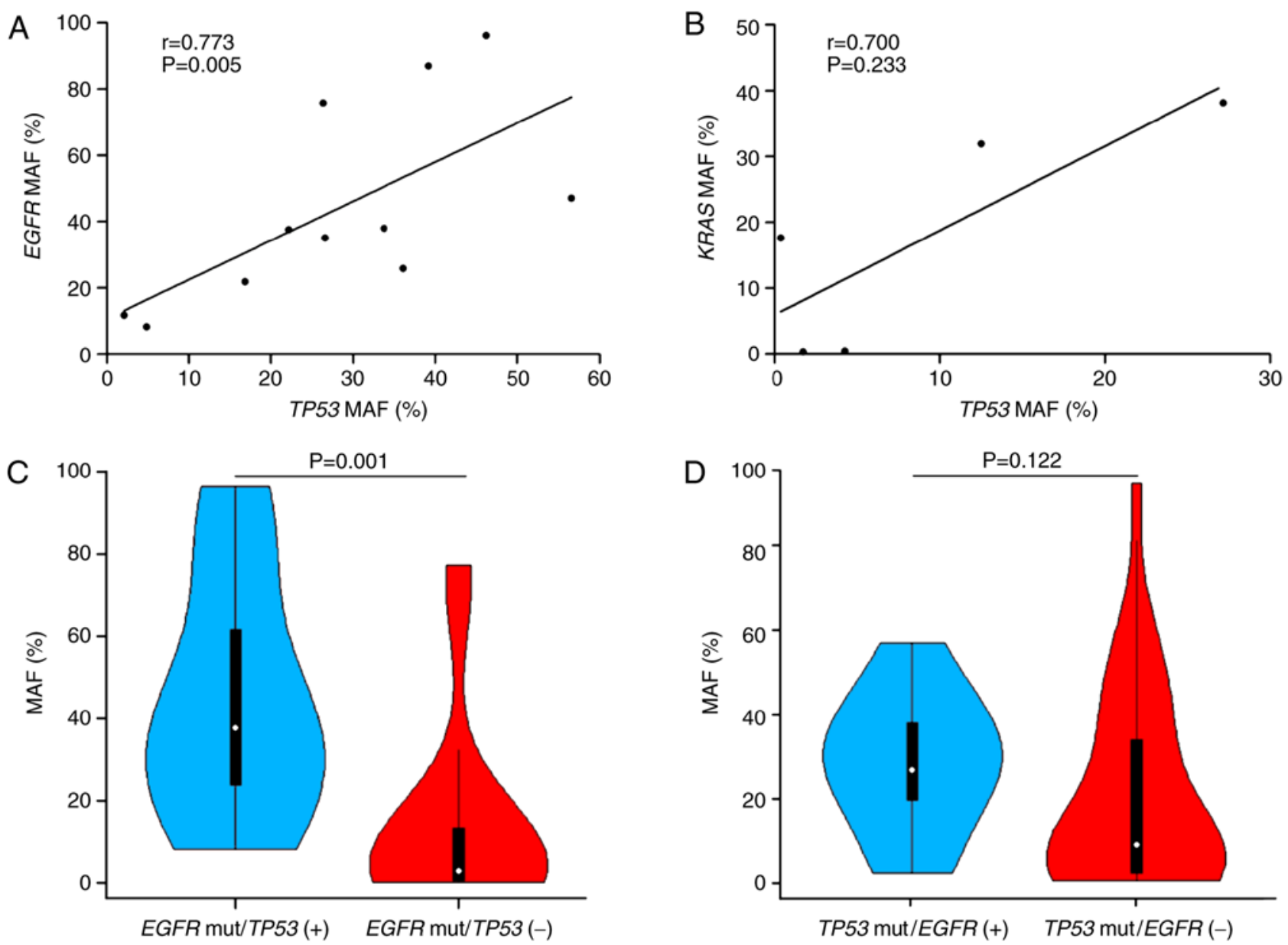

Figure 3. Comprehensive analysis of the MAFs of TP53 and EGFR in non-small cell lung cancer. (A) Scatter plot of the TP53 MAF vs. the EGFR MAF in the same patient. (B) Scatter plot of the TP53 MAF vs. the KRAS MAF in the same patient. (C) Violin plot of the difference between the EGFR MAF in patients with [EGFR mut/TP53 (+)] and without [EGFR mut/TP53 (-)] TP53 mutations. (D) Violin plot of the difference between the TP53 MAF in patients with [TP53 mut/EGFR (+)] and without [TP53 mut/EGFR (-)] EGFR mutations. MAF, mutant allele frequency; TP53, tumor protein 53; EGFR, epidermal growth factor receptor; KRAS, Kirseten rat sarcoma viral oncogene homolog; mut, mutant.

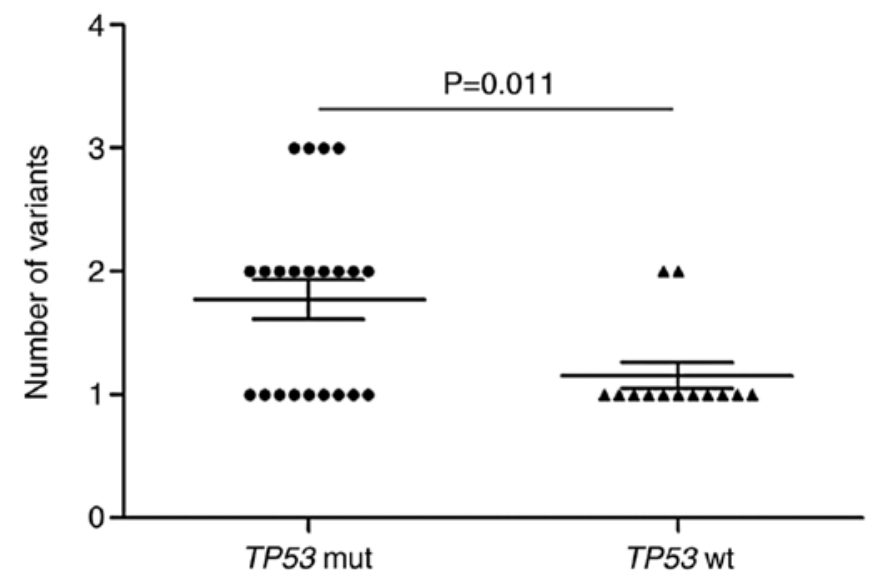

Figure 4. Comparison of the number of variants between the TP53 mutan (mut) and the wild-type baseline tumors in patients with non-small cell lung cancer. Variants include single nucleotide variants and small insertions or deletions. TP53, tumor protein 53; mut, mutant; wt, wild-type.

ctDNA-detectable mutations was significantly associated with clinical stage $(\mathrm{P}=0.014)$; however, no significant association was observed between other clinical features (such as age, sex, histological type and smoking history) and ctDNA-detectable mutation frequency (Table VI).

Among the patients with plasma ctDNA samples, the levels of tumor serum markers (such as CA242, NSE, CEA, CA125 and $\mathrm{CA} 15-3$ ) were determined in 38 patients. Further analysis revealed that CA242 levels in patients with ctDNA-detectable mutations were significantly higher than those observed in patients without ctDNA-detectable mutations $(\mathrm{P}=0.034$; Fig. 5). There was no significant difference in the levels of other tumor serum markers between patients with and without ctDNA-detectable mutations.

\section{Discussion}

Through NGS, the present study comprehensively analyzed genomic alterations in tissue and plasma ctDNA samples derived from Chinese patients with NSCLC. In the present study, the associations between the frequencies of several driver gene alterations and clinical characteristics were observed. Of note, TP53 alterations were associated with SC, ERBB2 alterations were associated with smoking, and KRAS alterations seemed to primarily arise in male or smoking patients with NSCLC. In addition, ERBB2 alterations, including somatic 
Table VI. Analysis between frequency of ctDNA-detectable mutations and clinicopathological characteristics.

\begin{tabular}{lcc}
\hline Characteristic & $\begin{array}{c}\text { Frequency of ctDNA- } \\
\text { detectable mutations, } \%\end{array}$ & P-value \\
\hline Sex & & \\
Male & 57.4 & 0.098 \\
Female & 83.3 & \\
Age, years & & 0.515 \\
$\quad \leq 60$ & 58.1 & \\
$>60$ & 66.7 & 0.712 \\
Histological type & & \\
AD & 70.0 & \\
SC & 65.4 & 0.915 \\
Smoking & & \\
Yes & 65.0 & 0.014 \\
No & 66.7 & \\
Stage & & \\
I+II+III & 40.0 & \\
IV & 75.7 & \\
\hline
\end{tabular}

ctDNA, circulating tumor DNA; AD, lung adenocarcinoma; SC, lung squamous cell carcinoma.

mutations and CNVs, were only encountered in patients exhibiting metastasis, implying that aberrations in ERBB2 may occur later during NSCLC progression. The differences in the prevalence of several driver gene mutations (such as EGFR, ERBB2 and TP53) between Chinese and TCGA cohorts was indicative of the specificity of the gene mutational spectrum in Chinese patients with NSCLC. Furthermore, although the frequency of targetable alterations in patients with $\mathrm{AD}$ was markedly higher than in patients with $\mathrm{SC}$, the data from the present study demonstrated that a proportion of patients with SC could potentially benefit from targeted therapy and that the detection of genomic alterations in patients with $\mathrm{SC}$ is therefore required.

Multiple resistance mechanisms against first-generation EGFR-TKI treatment, including EGFR amplification, the EGFR T790M mutation and ERBB2 alterations, have been described previously (32). Based on the genomic landscape, the present study demonstrated that EGFR amplification coexisted with EGFR somatic mutations, but was mutually exclusive with EGFR T790M mutations; there were no cases of simultaneous ERBB2 somatic mutations and amplification. This implies that patients with NSCLC may not exhibit mutations and amplification of the same genes in resistance to EGFR-TKI. Further analysis revealed that an EGFR mutation was seen alongside each other gene (MET, ERBB2, KRAS and ALK) mutation in only one patient each, demonstrating the importance of multi-gene panel testing in NSCLC. However, no co-occurrence of EGFR alterations with RET or BRAF alterations was observed. The statistical analyses revealed that EGFR alterations were significantly mutually exclusive with TP53 or KRAS alterations and potentially mutually exclusive

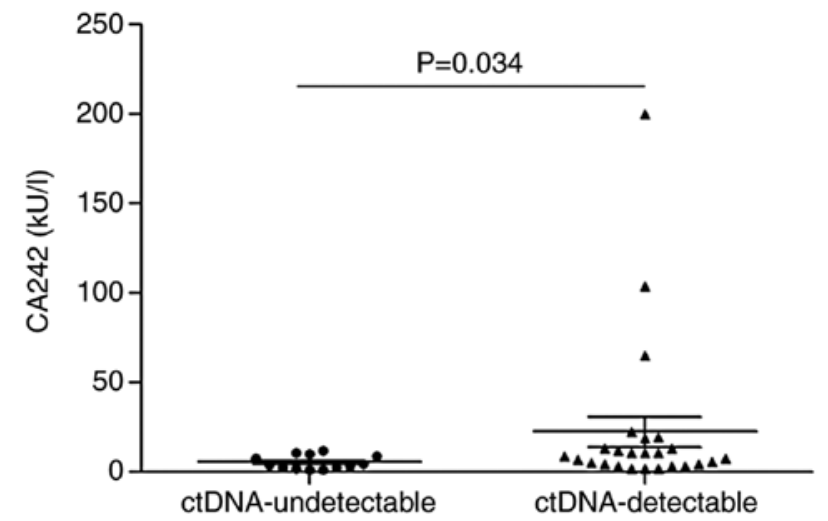

Figure 5. Comparison of CA242 levels between patients with non-small cell lung cancer with ctDNA-undetectable and ctDNA-detectable mutations. ctDNA, circulating tumor DNA.

with ERBB2 alterations. These findings are indicative of mutual exclusivity between EGFR alterations and alterations in other driver genes in NSCLC.

A previous study reported that EGFR and TP53 are the two dominant clonal mutant genes in NSCLC (26). The significant linear correlation between the MAFs of TP53 and EGFR in individual patients further confirmed that TP53 and EGFR mutations regularly co-occur as clonal events in the evolution of NSCLC. Notably, marked differences in the EGFR MAFs of patients with and without TP53 mutations were observed. A recent study suggested that the abundance of EGFR-activating mutations was correlated with the efficacy of EGFR-TKIs in advanced NSCLC (33). According to the results of the present study, there may be differences in the therapeutic effect of EGFR-TKIs in patients with EGFR-activating mutations with and without TP53 mutations. Another finding was the difference in the number of mutations among baseline tumors with and without TP53 mutations. Several studies have reported an association between the number of mutations and prognosis, or between mutations in specific genes and prognosis (27-31). As the present study did not obtain complete information on the therapeutic effect and prognosis in a large proportion of patients, determination of the efficacy of EGFR-TKIs and prognosis in tumors with and without TP53 mutations could not be assessed.

At present, the detection of genomic alterations primarily relies on the analysis of cancer tissues obtained via surgical excision or biopsy. However, tumor tissues are not always available for all patients, and tissue biopsy of a single site may not fully reflect the tumor genomic landscape due to tumor genetic heterogeneity (34). Therefore, in the present study, the use of plasma ctDNA was assessed for patients without sufficient tissue samples to screen for genomic alterations to guide personalized therapy. In the analysis of the ctDNA and tissue profiles, the landscape and frequencies of genomic alterations in ctDNA samples demonstrated a strong similarity with those observed in tissue samples, supporting the feasibility and use of plasma ctDNA testing among patients with NSCLC. The association between the frequency of plasma ctDNA-detectable mutations and clinical characteristics was also assessed in the present study, which revealed that the frequency of ctDNA-detectable 
mutations was significantly associated with clinical stage, consistent with previous results (15). In addition, it was observed that serum CA242 levels were significantly higher in patients with ctDNA-detectable mutations when compared with patients without ctDNA-detectable mutations. A prior study reported that serum CA242 levels were associated with clinical stage in NSCLC (35). Therefore, the present study provides a novel line of evidence confirming the prior result that ctDNA-detectable mutations are associated with clinical stage in NSCLC.

In conclusion, the present study identified targetable genetic alterations in $50.5 \%$ of patients with NSCLC using targeted NGS and discovered significant correlations between the frequency of genomic variants in tissue and plasma ctDNA samples. In addition, the results of the present study demonstrated that the MAF of EGFR was associated with that of TP53 in individual tumors and that the EGFR MAF in patients with the TP53 mutation was different to that observed in patients without the TP53 mutation. Although further studies are required to confirm these findings, these findings provide an improved understanding of the spectra of genomic alterations detected by tissue and plasma ctDNA assays in Chinese patients with NSCLC.

\section{Acknowledgements}

The abstract was presented at the 55th Meeting of ASCO, 31 May to 4 June, 2019 in Chicago, USA and published as abstract no. e20534 in Journal of Clinical Oncology, Vol. 37: 15_suppl.

\section{Funding}

The present study was funded by the Scientific Research Fund Project in the Department of Science and Technology of Hunan Province, China (grant. no. 2014SK3006).

\section{Availability of data and materials}

All data analyzed during the present study are included in this published article.

\section{Authors' contributions}

HY, HC, HW, SC and JC conceived and designed the project. HY, JZ, LZ, XW, YL, DY and TC collected samples and performed the experiments; HC, HW, FL, XL and JG analyzed the data. HY, HC, HW, SC and JC wrote the paper. All authors reviewed and edited the manuscript, and approved the final version for publication.

\section{Ethics approval and consent to participate}

The present study was approved by the Committee of Medical Ethics of Hunan Cancer Hospital (Changsha, China). All patients provided written informed consent prior to the study start.

\section{Patient consent for publication}

Not applicable.

\section{Competing interests}

The authors declare that they have no competing interests.

\section{References}

1. Liu K, Chen HL, Wang S, Gu MM, Chen XM, Zhang SL, Yu KJ and You QS: High expression of RIOK2 and NOB1 predict human non-small cell lung cancer outcomes. Sci Rep 6: 28666, 2016.

2. Yang J, Lin J, Liu T, Chen T, Pan S, Huang W and Li S: Analysis of lncRNA expression profiles in non-small cell lung cancers (NSCLC) and their clinical subtypes. Lung Cancer 85: 110-115, 2014.

3. Torr LA, Bray F, Siegel RL, Ferlay J, Lortet-Tieulent J and Jemal A: Global cancer statistics, 2012. CA Cancer J Clin 65: 87-108, 2015

4. Tursz T, Andre F, Lazar V, Lacroix L and Soria JC: Implications of personalized medicine-perspective from a cancer center. Nat Rev Clin Oncol 8: 177-183, 2011.

5. Swanton $\mathrm{C}$ and Govindan R: Clinical implications of genomic discoveries in lung cancer. N Engl J Med 374: 1864-1873, 2016.

6. Berge EM and Doebele RC: Targeted therapies in non-small cell lung cancer: Emerging oncogene targets following the success of epidermal growth factor receptor. Semin Oncol 41: 110-125, 2014.

7. Travis WD, Brambilla E, Nicholson AG, Yatabe Y, Austin JHM, Beasley MB, Chirieac LR, Dacic S, Duhig E, Flieder DB, et al: The 2015 world health organization classification of lung tumors: Impact of genetic, clinical and radiologic advances since the 2004 classification. J Thorac Oncol 10: 1243-1260, 2015.

8. Vargas AJ and Harris CC: Biomarker development in the precision medicine era: Lung cancer as a case study. Nat Rev Cancer 16: 525-537, 2016.

9. Tsao AS, Scagliotti GV, Bunn PA Jr, Carbone DP, Warren GW, Bai C, de Koning HJ, Yousaf-Khan AU, McWilliams A, Tsao MS, et al: Scientific advances in lung cancer 2015. J Thorac Oncol 11: 613-638, 2016.

10. Shyr D and Liu Q: Next generation sequencing in cancer research and clinical application. Biol Proced Online 15: 4, 2013.

11. Gagan J and Van Allen EM: Next-generation sequencing to guide cancer therapy. Genome Med 7: 80, 2015.

12. Gerlinger M, Rowan AJ, Horswell S, Math M, Larkin J, Endesfelder D, Gronroos E, Martinez P, Matthews N, Stewart A, et al: Intratumor heterogeneity and branched evolution revealed by multiregion sequencing. N Engl J Med 366: 883-892, 2012.

13. Kris MG, Johnson BE, Berry LD, Kwiatkowski DJ, Iafrate AJ, Wistuba II, Varella-Garcia M, Franklin WA, Aronson SL, $\mathrm{Su} \mathrm{PF}$, et al: Using multiplexed assays of oncogenic drivers in lung cancers to select targeted drugs. JAMA 311: 1998-2006, 2014.

14. Crowley E, Di Nicolantonio F, Loupakis F and Bardelli A: Liquid biopsy: Monitoring cancer-genetics in the blood. Nat Rev Clin Oncol 10: 472-484, 2013.

15. Bettegowda C, Sausen M, Leary RJ, Kinde I, Wang Y, Agrawal N, Bartlett BR, Wang H, Luber B, Alani RM, et al: Detection of circulating tumor DNA in early- and late-stage human malignancies. Sci Transl Med 6: 224ra24, 2014.

16. Hicks JK, Saller J, Wang E, Boyle T and Gray JE: Cell-free circulating tumor DNA supplementing tissue biopsies for identification of targetable mutations: Implications for precision medicine and considerations for reconciling results. Lung Cancer 111: 135-138, 2017.

17. Xu S, Lou F, Wu Y, Sun DQ, Zhang JB, Chen W, Ye H, Liu JH, Wei S, Zhao MY, et al: Circulating tumor DNA identified by targeted sequencing in advanced-stage non-small cell lung cancer patients. Cancer Lett 370: 324-331, 2016.

18. Pisapia P, Pepe F, Smeraglio R, Russo M, Rocco D, Sgariglia R, Nacchio M, De Luca C, Vigliar E, Bellevicine C, et al: Cell free DNA analysis by $\mathrm{SiRe}^{\circledR}$ next generation sequencing panel in non small cell lung cancer patients: Focus on basal setting. J Thorac Dis 9 (Suppl 13): S1383-S1390, 2017.

19. Mayo-de-Las-Casas C, Jordana-Ariza N, Garzón-Ibañez M, Balada-Bel A, Bertrán-Alamillo J, Viteri-Ramírez S, Reguart N, Muñoz-Quintana MA, Lianes-Barragan P, Camps C, et al: Large scale, prospective screening of EGFR mutations in the blood of advanced NSCLC patients to guide treatment decisions. Ann Oncol 28: 2248-2255, 2017. 
20. Chen K, Zhang J, Guan T, Yang F, Lou F, Chen W, Zhao M, Zhang J, Chen S and Wang J: Comparison of plasma to tissue DNA mutations in surgical patients with non-small cell lung cancer. J Thorac Cardiovasc Surg 154: 1123-1131.e2, 2017.

21. Kimura $\mathrm{K}$ and Koike A: Ultrafast SNP analysis using the Burrows-Wheeler transform of short-read data. Bioinformatics 31: $1577-1583,2015$.

22. McKenna A, Hanna M, Banks E, Sivachenko A, Cibulskis K Kernytsky A, Garimella K, Altshuler D, Gabriel S, Daly M and DePristo MA: The genome analysis toolkit: A MapReduce framework for analyzing next-generation DNA sequencing data. Genome Res 20: 1297-1303, 2010.

23. Cibulskis K, Lawrence MS, Carter SL, Sivachenko A, Jaffe D, Sougnez C, Gabriel S, Meyerson M, Lander ES and Getz G: Sensitive detection of somatic point mutations in impure and heterogeneous cancer samples. Nat Biotechnol 31: 213-219, 2013.

24. Li J, Lupat R, Amarasinghe KC, Thompson ER, Doyle MA Ryland GL, Tothill RW, Halgamuge SK, Campbell IG and Gorringe KL: CONTRA: Copy number analysis for targeted resequencing. Bioinformatics 28: 1307-1313, 2012.

25. Campbell JD, Alexandrov A, Kim J, Wala J, Berger AH, Pedamallu CS, Shukla SA, Guo G, Brooks AN, Murray BA, et al: Distinct patterns of somatic genome alterations in lung adenocarcinomas and squamous cell carcinomas. Nat Genet 48: 607-616, 2016.

26. Jamal-Hanjani M, Wilson GA, McGranahan N, Birkbak NJ, Watkins TBK, Veeriah S, Shafi S, Johnson DH, Mitter R, Rosenthal R, et al: Tracking the evolution of non-small cell lung cancer. N Engl J Med 376: 2109-2121, 2017.

27. Thompson JC, Yee SS, Troxel AB, Savitch SL, Fan R, Balli D, Lieberman DB, Morrissette JD, Evans TL, Bauml J, et al: Detection of therapeutically targetable driver and resistance mutations in lung cancer patients by next-generation sequencing of cell-free circulating tumor DNA. Clin Cancer Res 22: 5772-5782, 2016.

28. Nahar R, Zhai W, Zhang T, Takano A, Khng AJ, Lee YY, Liu X, Lim $\mathrm{CH}$, Koh TPT, Aung ZW, et al: Elucidating the genomic architecture of Asian EGFR-mutant lung adenocarcinoma through multi-region exome sequencing. Nat Commun 9: 216, 2018.
29. VanderLaan PA, Rangachari D, Mockus SM, Spotlow V, Reddi HV, Malcolm J, Huberman MS, Joseph LJ, Kobayashi SS and Costa DB: Mutations in TP53, PIK3CA, PTEN and other genes in EGFR mutated lung cancers: Correlation with clinical outcomes. Lung Cancer 106: 17-21, 2017.

30. Molina-Vila MA, Bertran-Alamillo J, Gascó A, Mayo-de-las-Casas C, Sánchez-Ronco M, Pujantell-Pastor L, Bonanno L, Favaretto AG, Cardona AF, Vergnenègre A, et al: Nondisruptive p53 mutations are associated with shorter survival in patients with advanced non-small cell lung cancer. Clin Cancer Res 20: 4647-4659, 2014.

31. Mascaux C, Iannino N, Martin B, Paesmans M, Berghmans T, Dusart M, Haller A, Lothaire P, Meert AP, Noel S, et al: The role of RAS oncogene in survival of patients with lung cancer: A systematic review of the literature with meta-analysis. $\mathrm{Br}$ J Cancer 92: 131-139, 2005.

32. Rotow $\mathbf{J}$ and Bivona TG: Understanding and targeting resistance mechanisms in NSCLC. Nat Rev Cancer 17: 637-658, 2017.

33. Li X, Cai W, Yang G, Su C, Ren S, Zhao C, Hu R, Chen X, Gao G, Guo Z, et al: Comprehensive analysis of EGFR-mutant abundance and its effect on efficacy of EGFR TKIs in advanced NSCLC with EGFR mutations. J Thorac Oncol 12: 1388-1397, 2017.

34. Sholl LM, Aisner DL, Varella-Garcia M, Berry LD, Dias-Santagata D, Wistuba II, Chen H, Fujimoto J, Kugler K, Franklin WA, et al: Multi-institutional oncogenic driver mutation analysis in lung adenocarcinoma: The lung cancer mutation consortium experience. J Thorac Oncol 10: 768-777, 2015.

35. Pujol JL, Cooper EH, Lehmann M, Purves DA, Dan-Aouta M, Midander J, Godard P and Michel FB: Clinical evaluation of serum tumor marker CA 242 in non-small cell lung cancer. $\mathrm{Br}$ J Cancer 67: 1423-1429, 1993.

This work is licensed under a Creative Commons Attribution-NonCommercial-NoDerivatives 4.0 International (CC BY-NC-ND 4.0) License. 